\title{
Kinetic Modeling of Ethylene Epoxidation Kinetics as a Function of Chlorine Coverage over a Highly Promoted Silver Catalyst
}

\author{
James W. Harris, ${ }^{1}$ Le Wang, ${ }^{2}$ and Joseph F. DeWilde ${ }^{2 *}$ \\ 1. Department of Chemical Engineering and Materials Science, University of Minnesota, \\ 421 Washington Ave SE, Minneapolis, MN 55455, USA \\ 2. Dow, Inc., Midland, MI, 48640 \\ *Corresponding author. E-mail: jfdewilde@dow.com
}

\section{SUPPORTING INFORMATION}

\section{S.1 Parameter Values and Quality of Regression for Each Model.}

Table S.1 compares the goodness of fit between the models considered in this study. Values of the Mean Absolute Relative Error (MARD) and Akaike Information Criterion (AIC), normalized by number of observations, for each model are reported in Table S.1. The AIC rewards goodness-offit while penalizing overfitting, and the minimum value of the AIC represents the model that best fits the data with the least overfitting. As shown in Table S.1, Model D has the minimum AIC of the models considered here.

Table S.1. Comparison of goodness-of-fit parameters for Models A-F.

\begin{tabular}{ccc}
\hline Model & MARD & $\begin{array}{c}\text { AIC/\# of } \\
\text { observations }\end{array}$ \\
\hline A & $19.30 \%$ & -26.84 \\
B & $21.31 \%$ & -26.50 \\
C & $17.85 \%$ & -26.76 \\
D & $18.18 \%$ & -26.97 \\
E & $16.67 \%$ & -26.53 \\
F & $15.06 \%$ & -26.75 \\
\hline
\end{tabular}




\section{S.1.1 Model A.}

Table S.2 Values for regressed parameters from Model A. Parameters $b_{1}$ and $b_{2}$ are not fitted in this model by definition.

\begin{tabular}{ccccc}
\hline Parameter & Value & Uncertainty & State & Unit \\
\hline $\ln K_{1,0}$ & -7.26 & \pm 2.02 & Fitted & - \\
$b_{1}$ & 0 & & Not Fitted & - \\
$\ln K_{2,0}$ & 1.80 & N/A & Fitted & - \\
$b_{2}$ & 0 & & Not Fitted & - \\
$\ln K_{3,0}$ & -0.090 & \pm 2.56 & Fitted & - \\
$b_{3}$ & 0.488 & \pm 0.709 & Fitted & - \\
$\ln K_{4}$ & 7.03 & \pm 0.301 & Fitted & N \\
$\ln k_{5}$ & 6.71 & N/A & Fitted & $\ln \left(\left[\mathrm{g}_{\text {cat }} \mu \mathrm{mol}^{-1} \mathrm{~s}^{-1}\right]\right)$ \\
$\ln \left(\frac{k_{7}}{k_{6}}\right)_{0}$ & -0.891 & \pm 0.32 & Fitted & - \\
$a_{7}$ & 2.53 & \pm 7.27 & Fitted & - \\
$b_{7}$ & -3.47 & \pm 3.04 & Fitted & - \\
\hline
\end{tabular}
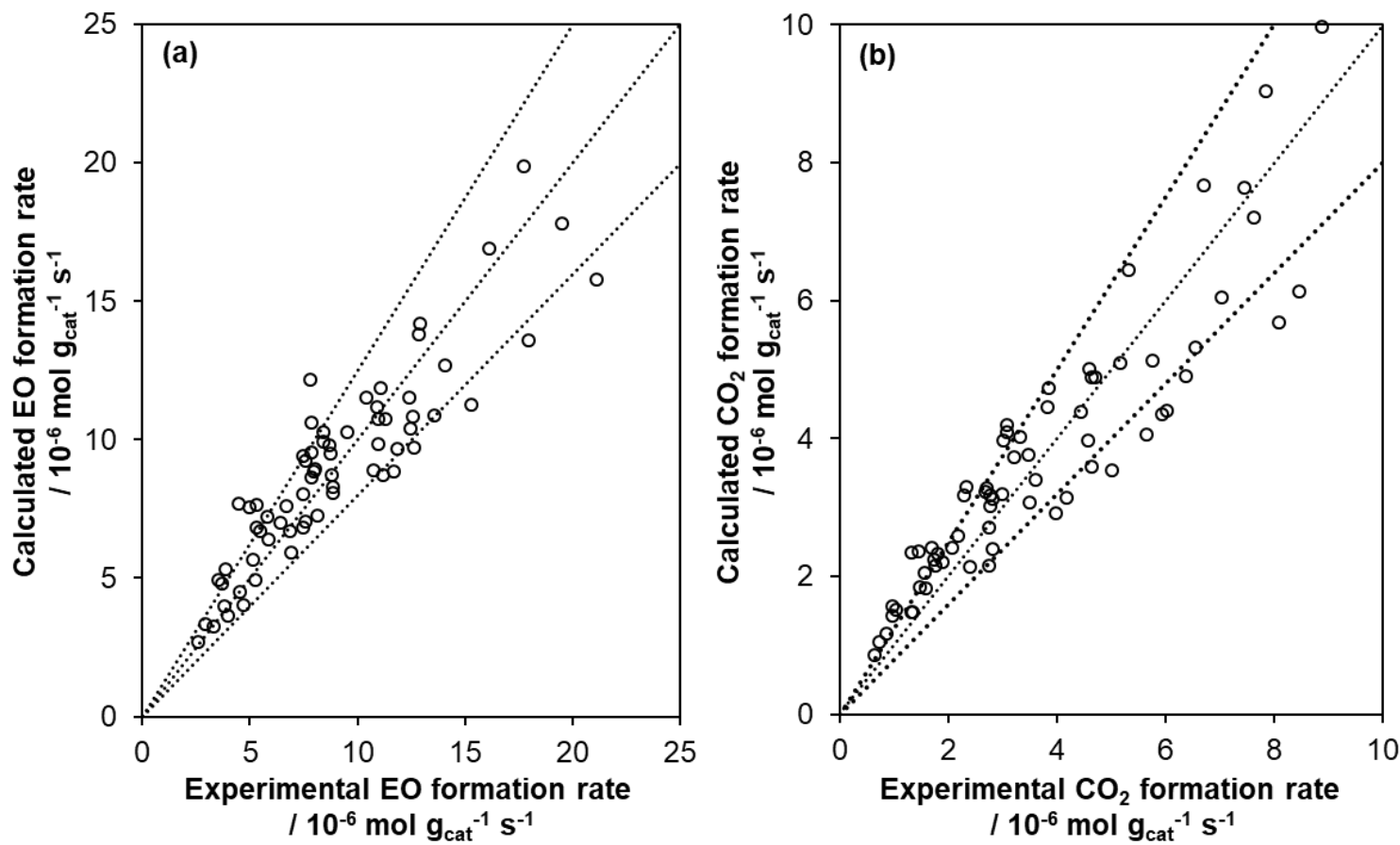

Figure S.1 Parity plot for all experimental data (circles) using predictions from Model A for (a) $\mathrm{EO}$ formation rates and (b) $\mathrm{CO}_{2}$ formation rates. Dashed lines show parity and $20 \%$ error bounds. Experimental uncertainty in measured reaction rates are $\pm 20 \%$ of the reported values. Experimental data were previously reported in Harris and Bhan ${ }^{1}$ and Chen et al. ${ }^{2}$ 


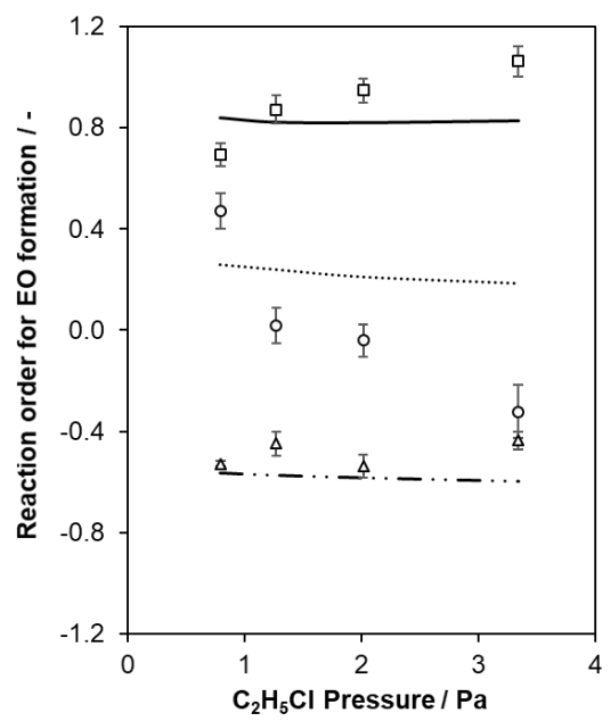

Figure S.2 Comparison of experimentally measured apparent reaction orders (symbols) with reaction orders predicted from Model A (lines) for dioxygen (squares, solid line), ethylene (circles, dashed line), and carbon dioxide (triangles, dashed-dotted line). Experimental uncertainty in measured reaction rates are $\pm 20 \%$ of the reported values. Experimental data were previously reported in Harris and Bhan ${ }^{1}$ and Chen et al. ${ }^{2}$

S.1.2 Model B.

Table S.3 Values for regressed parameters in Model B. Parameters $b_{2}$ and $b_{3}$ are not fitted in Model B by definition.

\begin{tabular}{ccccc}
\hline Parameter & Value & Uncertainty & State & Unit \\
\hline $\ln K_{1,0}$ & 0.174 & \pm 2.04 & Fitted & - \\
$b_{1}$ & -0.0762 & \pm 0.669 & Fitted & - \\
$\ln K_{2,0}$ & -0.00993 & N/A & Fitted & - \\
$b_{2}$ & 0 & & Not Fitted & - \\
$\ln K_{3,0}$ & 1.72 & N/A & Fitted & - \\
$b_{3}$ & 0 & & Not Fitted & - \\
$\ln K_{4}$ & 3.94 & \pm 1.12 & Fitted & - \\
$\ln k_{5}$ & 2.63 & \pm 1.86 & Fitted & $\ln \left(\left[\mathrm{g}_{\text {cat }} \mu \mathrm{mol}^{-1} \mathrm{~s}^{-1}\right]\right.$ \\
$\ln \left(\frac{k_{7}}{k_{6}}\right)_{0}$ & -1.88 & \pm 0.676 & Fitted & - \\
$a_{7}$ & -8.32 & \pm 12.1 & Fitted & - \\
$b_{7}$ & 2.94 & \pm 5.70 & Fitted & - \\
\hline
\end{tabular}



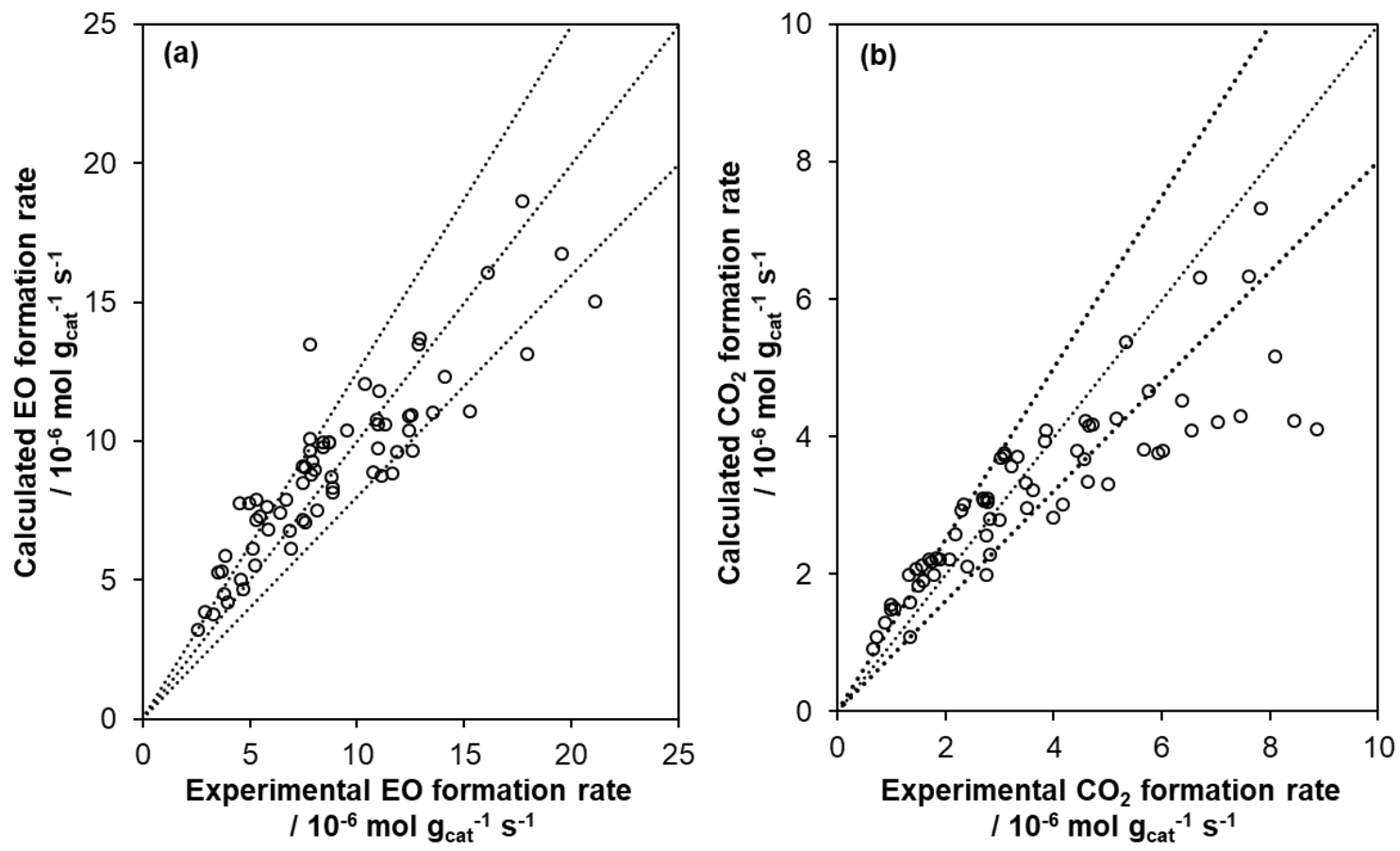

Figure S.3 Parity plot for all experimental data (circles) using predictions from Model B for (a) $\mathrm{EO}$ formation rates and (b) $\mathrm{CO}_{2}$ formation rates. Experimental uncertainty in measured reaction rates are $\pm 20 \%$ of the reported values. Experimental data were previously reported in Harris and Bhan ${ }^{1}$ and Chen et al. ${ }^{2}$ Dashed lines show parity and $20 \%$ error bounds.

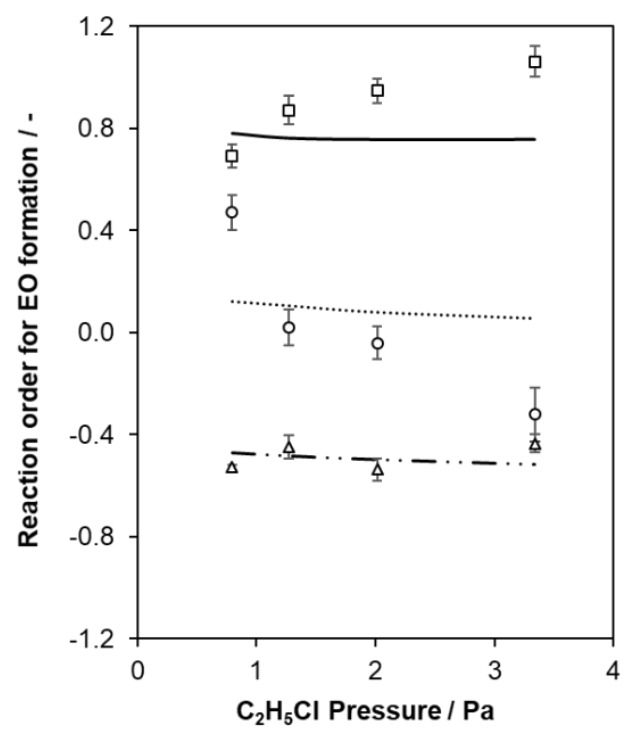

Figure S.4 Comparison of experimentally measured apparent reaction orders (symbols) with reaction orders predicted from Model B (lines) for dioxygen (squares, solid line), ethylene (circles, dashed line), and carbon dioxide (triangles, dashed-dotted line). 


\section{S.1.3 Models C and D.}

An alternate treatment for varying the ratio of $\mathrm{k}_{7}: \mathrm{k}_{6}$ with a linear dependence on chlorine coverage was considered:

$$
\frac{k_{7}}{k_{6}}=\left(\frac{k_{7}}{k_{6}}\right)_{0} \exp \left(b_{6} \theta_{C l}\right)
$$

The performance of this linear version of Model D (Eq. S.1) is very similar to that of quadratic version (Eq. 21). However, the linear version was rejected based on slightly worse description of selectivity (Figure S.5) and larger value of Akaike Information Criterion (AIC) (Table S.5).

Table S.4. Comparison of goodness-of-fit for Model D with linear and quadratic dependencies of the ratio $\mathrm{k}_{7}: \mathrm{k}_{6}$ on $\theta_{\mathrm{Cl}}$.

\begin{tabular}{ccc}
\hline Model & MARD & $\begin{array}{c}\text { AIC/\# of } \\
\text { observations }\end{array}$ \\
\hline Linear & $17.93 \%$ & -26.95 \\
Quadratic & $18.18 \%$ & -26.97 \\
\hline
\end{tabular}
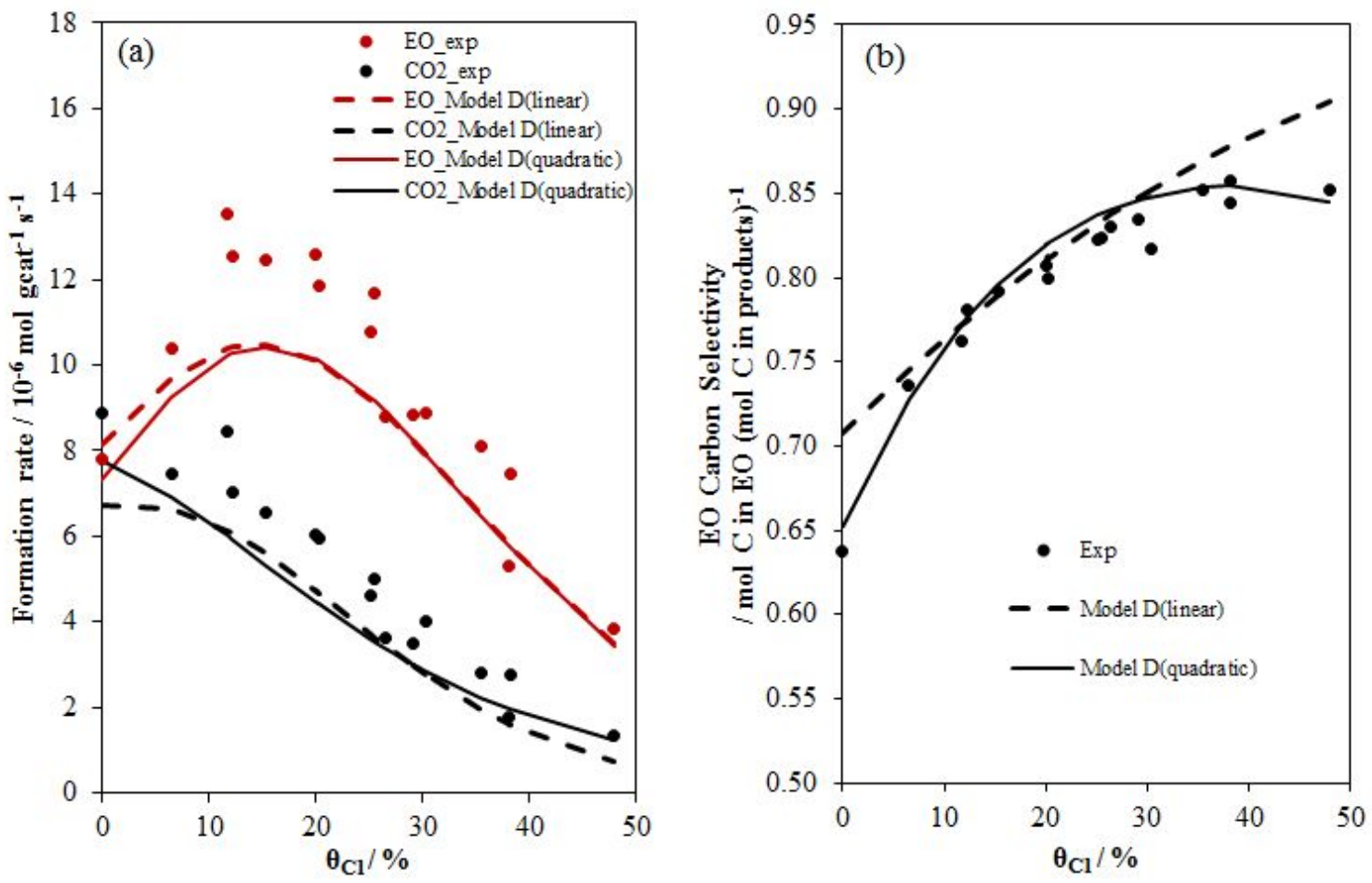

Figure S.5. Trends in (a) EO (red circles ) and $\mathrm{CO}_{2}$ (black circles) rates and (b) EO selectivity (black circles) as a function of $\mathrm{Cl}$ coverage comparing Model $\mathrm{C}$ with experimental data (circles) using either a linear dependence of $\mathrm{k}_{7}: \mathrm{k}_{6}$ on $\mathrm{Cl}$ coverage (dashed black lines) or a quadratic dependence on $\mathrm{Cl}$ coverage (solid black lines). Experimental conditions: $159 \mathrm{kPa}$ ethylene, 15.9 $\mathrm{kPa}$ dioxygen, $5.3 \mathrm{kPa}$ carbon dioxide, $0.53-74.2 \mathrm{kPa}$ ethane, 0-3.3 Pa ethyl chloride, balance He; total pressure, $530 \mathrm{kPa}$; temperature, $513 \mathrm{~K} ; \mathrm{SV}, 500,000 \mathrm{~h}^{-1}$. Experimental uncertainty in $\mathrm{Cl}$ coverages are $\pm 21 \%$ of the reported values, and uncertainties in measured reaction rates are $\pm 20 \%$ of the reported values. Adapted from Harris and Bhan. ${ }^{1}$ 
Table S.5 Values for regressed parameters in Model C. Parameter $b_{2}$ is not fitted in Model C by definition.

\begin{tabular}{ccclc}
\hline Parameter & Value & Uncertainty & State & Unit \\
\hline $\ln K_{1,0}$ & -0.307 & \pm 4.60 & Fitted & - \\
$b_{1}$ & -10.5 & \pm 9.75 & Fitted & - \\
$\ln K_{2,0}$ & -0.441 & N/A & Fitted & - \\
$b_{2}$ & 0 & & Not Fitted & - \\
$\ln K_{3,0}$ & 3.77 & \pm 4.73 & Fitted & - \\
$b_{3}$ & -12.8 & \pm 8.24 & Fitted & - \\
$\ln K_{4}$ & 4.99 & \pm 0.328 & Fitted & - \\
$\ln k_{5}$ & 5.05 & N/A & Fitted & $\ln \left(\left[\mathrm{g}_{\mathrm{cat}} \mu \mathrm{mol}^{-1} \mathrm{~s}^{-1}\right]\right)$ \\
$\ln \left(\frac{k_{7}}{k_{6}}\right)_{0}$ & -1.36 & \pm 0.662 & Fitted & - \\
$a_{7}$ & 0.482 & \pm 11.8 & Fitted & - \\
$b_{7}$ & -1.49 & \pm 5.56 & Fitted & - \\
\hline
\end{tabular}

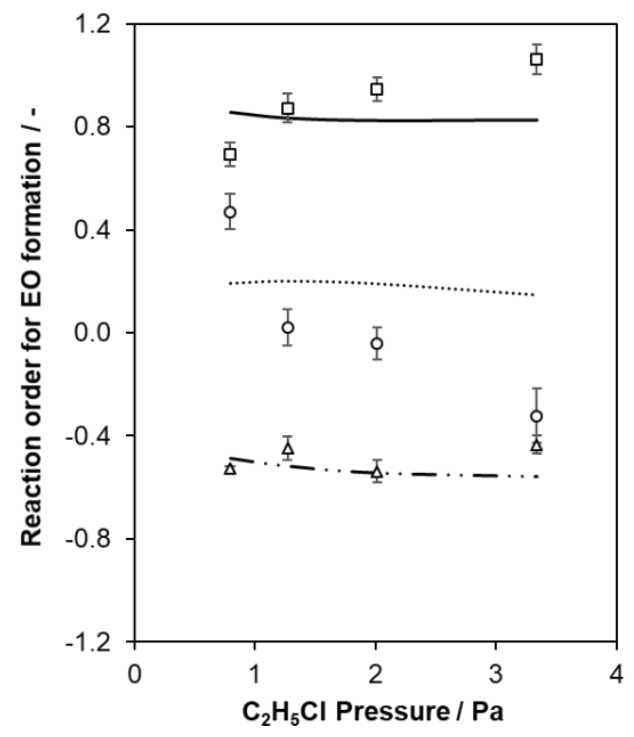

Figure S.6 Comparison of experimentally measured apparent reaction orders (symbols) with reaction orders predicted from Model C (lines) for dioxygen (squares, solid line), ethylene (circles, dashed line), and carbon dioxide (triangles, dashed-dotted line). 

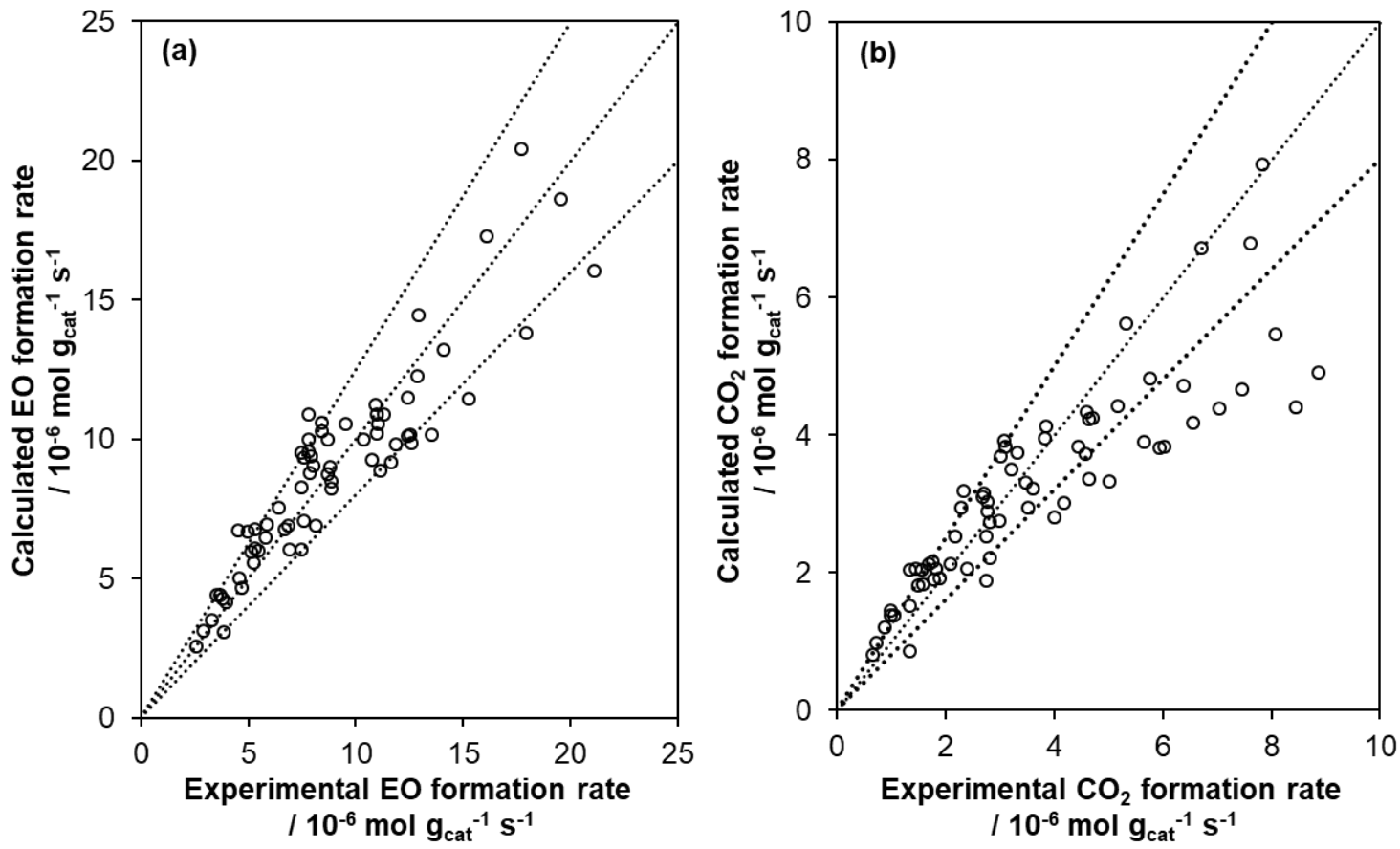

Figure S.7 Parity plot for all experimental data (circles) using predictions from Model C for (a) $\mathrm{EO}$ formation rates and (b) $\mathrm{CO}_{2}$ formation rates. Dashed lines show parity and $20 \%$ error bounds. Experimental uncertainty in measured reaction rates are $\pm 20 \%$ of the reported values. Experimental data were previously reported in Harris and Bhan ${ }^{1}$ and Chen et al. ${ }^{2}$

Table S.6 Values for regressed parameters in Model D.

\begin{tabular}{ccclc}
\hline Parameter & Value & Uncertainty & State & Unit \\
\hline $\ln K_{1,0}$ & -17.3 & \pm 0.598 & Fitted & - \\
$b_{1}$ & -0.0490 & \pm 9.59 & Fitted & - \\
$\ln K_{2,0}$ & 0.78 & $\mathrm{~N} / \mathrm{A}$ & Fitted & - \\
$b_{2}$ & 6.73 & \pm 7.55 & Fitted & - \\
$\ln K_{3,0}$ & -2.86 & \pm 1.51 & Fitted & - \\
$b_{3}$ & 11.6 & \pm 4.01 & Fitted & - \\
$\ln K_{4}$ & 12.0 & \pm 1.02 & Fitted & - \\
$\ln k_{5}$ & 16.0 & $\mathrm{~N} / \mathrm{A}$ & Fitted & $\ln \left(\left[\mathrm{g}_{\mathrm{cat}} \mu \mathrm{mol}^{-1} \mathrm{~s}^{-1}\right]\right)$ \\
$\ln \left(\frac{k_{7}}{k_{6}}\right)_{0}$ & -0.630 & \pm 0.366 & Fitted & - \\
$a_{7}$ & 7.81 & \pm 8.04 & Fitted & - \\
$b_{7}$ & -5.97 & \pm 3.40 & Fitted & - \\
\hline
\end{tabular}




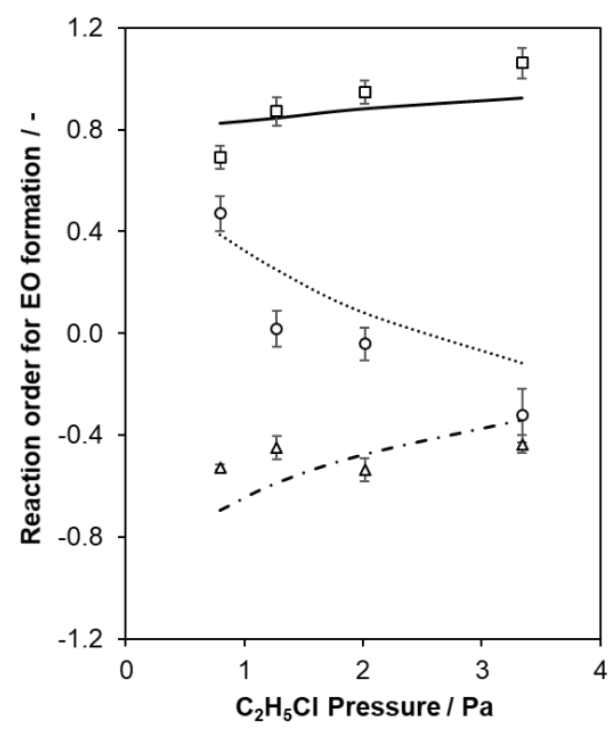

Figure S.8 Comparison of experimentally measured apparent reaction orders (symbols) with reaction orders predicted from Model D (lines) for dioxygen (squares, solid line), ethylene (circles, dashed line), and carbon dioxide (triangles, dashed-dotted line).
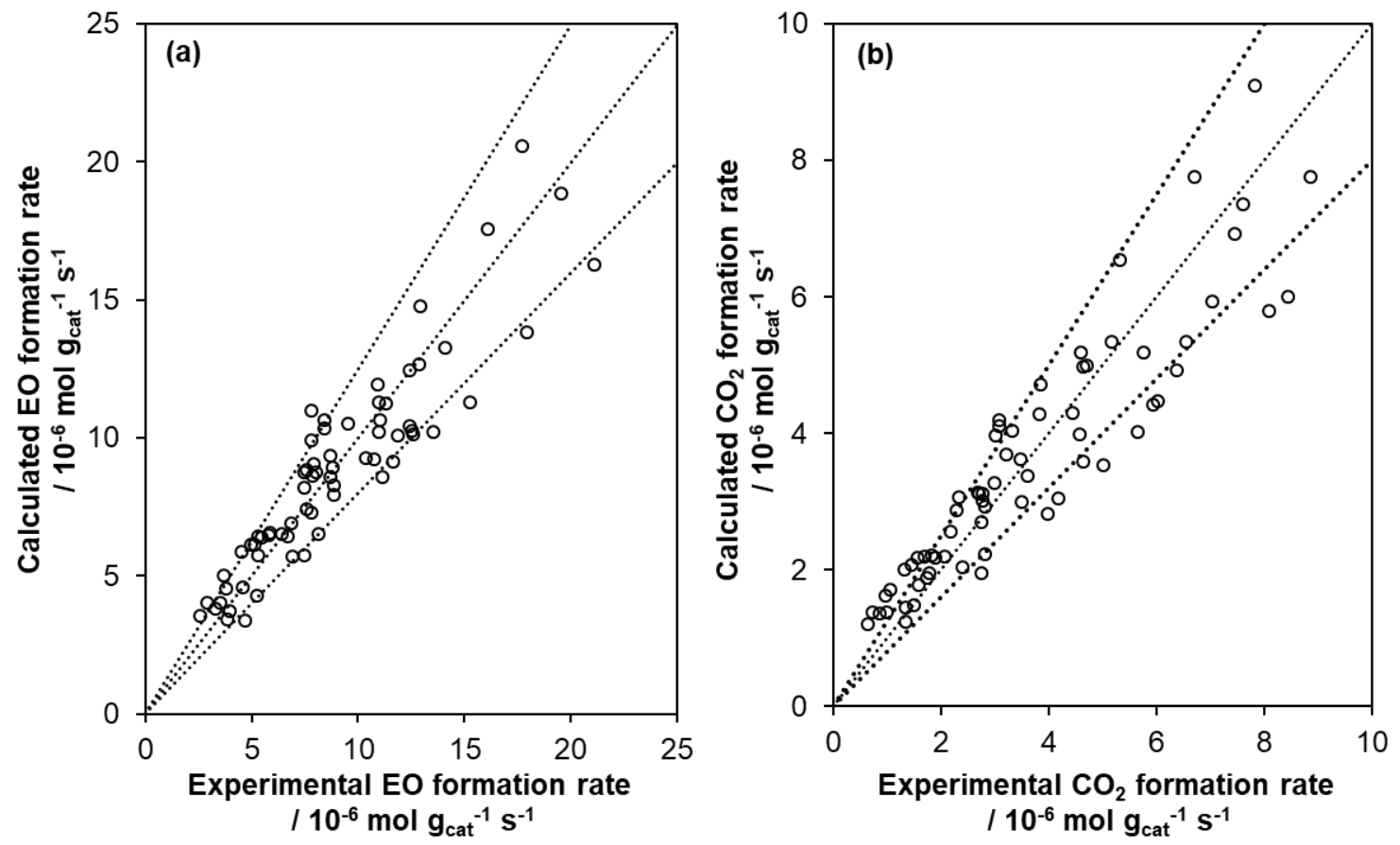

Figure S.9 Parity plot for all experimental data (circles) using predictions from Model D for (a) $\mathrm{EO}$ formation rates and (b) $\mathrm{CO}_{2}$ formation rates. Dashed lines show parity and 20\% error bounds. Experimental uncertainty in measured reaction rates are $\pm 20 \%$ of the reported values. Experimental data were previously reported in Harris and Bhan ${ }^{1}$ and Chen et al. ${ }^{2}$ 


\section{S.1.4 Model E.}

Table S.7 Values for regressed parameters in Model E. Parameters $a_{7}$ and $b_{7}$ are not fitted in Model E by definition.

\begin{tabular}{ccclc}
\hline Parameter & Value & Uncertainty & State & Unit \\
\hline $\ln K_{1,0}$ & -4.07 & \pm-2.00 & Fitted & - \\
$b_{1}$ & 7.42 & \pm 9.07 & Fitted & - \\
$\ln K_{2,0}$ & 0.53 & & Fitted & - \\
$b_{2}$ & 0.921 & \pm 3.32 & Fitted & - \\
$\ln K_{3,0}$ & -3.52 & \pm 2.01 & Fitted & - \\
$b_{3}$ & 13.2 & \pm 5.74 & Fitted & - \\
$\ln K_{4}$ & 4.37 & \pm 1.17 & Fitted & - \\
$\ln k_{5}$ & 3.17 & \pm 1.74 & Fitted & $\ln \left(\left[\mathrm{g}_{\text {cat }} \mu \mathrm{mol}^{-1} \mathrm{~s}^{-1}\right]\right)$ \\
$\ln \left(\frac{k_{7}}{k_{6}}\right)_{0}$ & 5.87 & \pm 0.996 & Fitted & $\ln \left(\left[\mathrm{g}_{\text {cat }} \mathrm{mmol}^{-1}\right]\right)$ \\
$a_{7}$ & 0 & & Not Fitted & - \\
$b_{7}$ & 0 & & Not Fitted & - \\
\hline
\end{tabular}
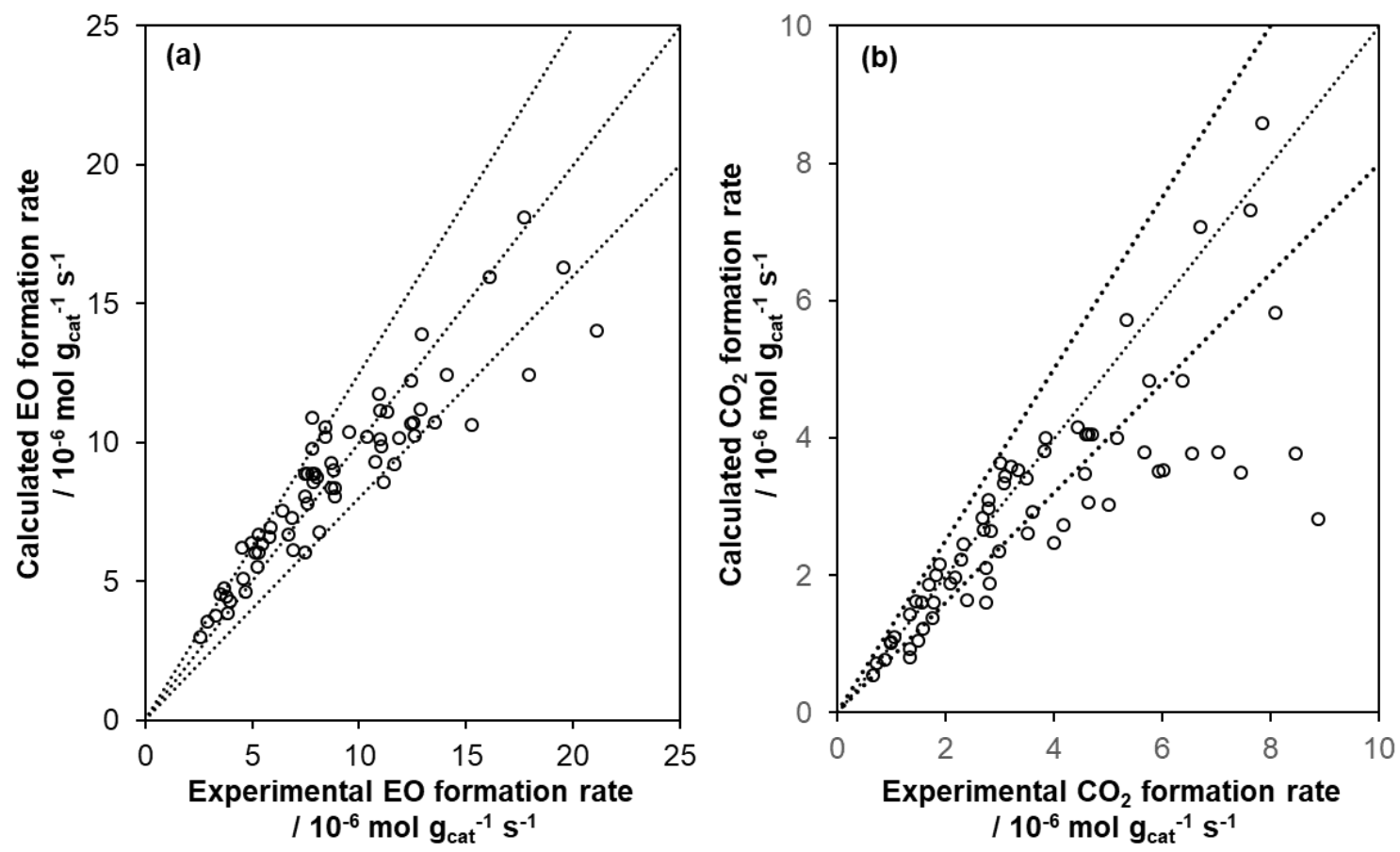

Figure S.10 Parity plot for all experimental data (circles) using predictions from Model E for (a) EO formation rates and (b) $\mathrm{CO}_{2}$ formation rates. Dashed lines show parity and $20 \%$ error bounds. Experimental uncertainty in measured reaction rates are $\pm 20 \%$ of the reported values. Experimental data were previously reported in Harris and Bhan ${ }^{1}$ and Chen et al. ${ }^{2}$ 


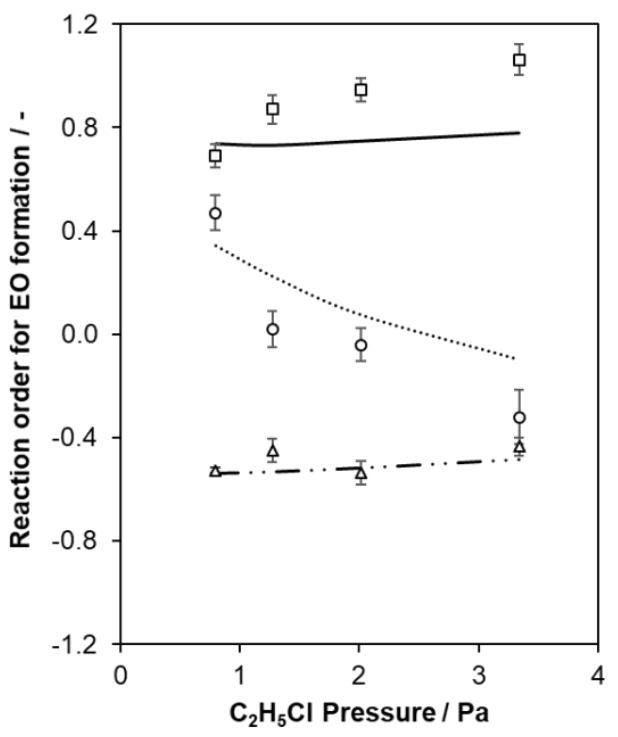

Figure S.11 Comparison of experimentally measured apparent reaction orders (symbols) with reaction orders predicted from Model E (lines) for dioxygen (squares, solid line), ethylene (circles, dashed line), and carbon dioxide (triangles, dashed-dotted line). Experimental uncertainty in measured reaction rates are $\pm 20 \%$ of the reported values. Experimental data were previously reported in Harris and Bhan ${ }^{1}$ and Chen et al. ${ }^{2}$ 


\section{S.1.5 Model F.}

Table S.8 Values for regressed parameters in Model F.

\begin{tabular}{ccclc}
\hline Parameter & Value & Uncertainty & State & Unit \\
\hline $\ln K_{1,0}$ & -18.0 & \pm 2.50 & Fitted & - \\
$b_{1}$ & 6.20 & \pm 9.25 & Fitted & - \\
$\ln K_{2,0}$ & 7.85 & \pm 2.70 & Fitted & - \\
$b_{2}$ & -1.69 & \pm 9.42 & Fitted & - \\
$\ln K_{3,0}$ & -4.69 & \pm 2.50 & Fitted & - \\
$b_{3}$ & 11.7 & \pm 3.73 & Fitted & - \\
$\ln K_{4}$ & 10.9 & \pm 6.51 & Fitted & - \\
$\ln k_{5}$ & 9.31 & $\mathrm{~N} / \mathrm{A}$ & Fitted & $\ln \left(\left[\mathrm{g}_{\text {cat }} \mu \mathrm{mol}^{-1} \mathrm{~s}^{-1}\right]\right)$ \\
$\ln \left(\frac{k_{7}}{k_{6}}\right)_{0}$ & 13.8 & \pm 1.43 & Fitted & $\ln \left(\left[\mathrm{g}_{\text {cat }} \mathrm{mmol}^{-1}\right]\right)$ \\
$a_{7}$ & 12.0 & \pm 8.58 & Fitted & - \\
$b_{7}$ & -7.77 & \pm 6.10 & Fitted & - \\
\hline
\end{tabular}
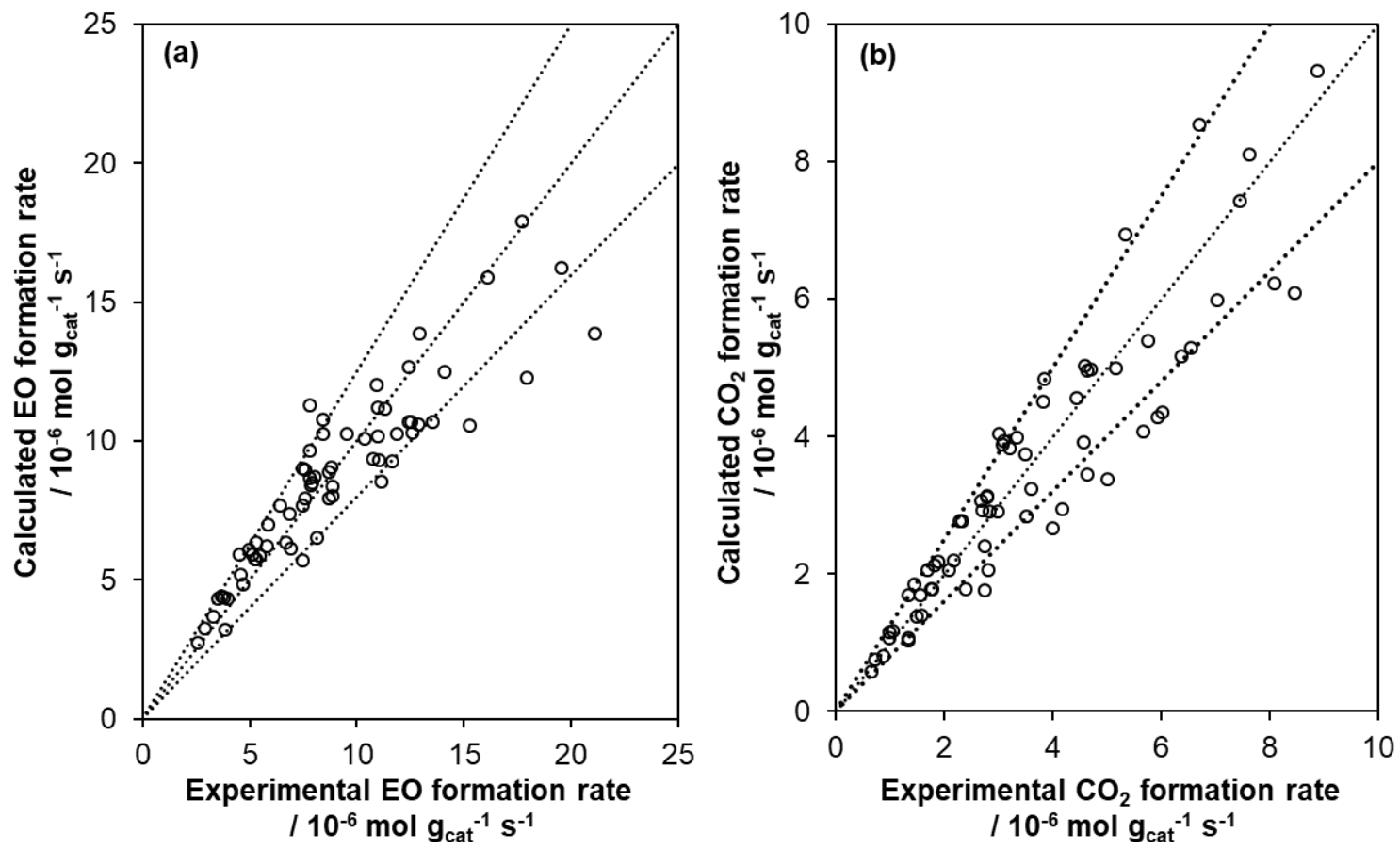

Figure S.12 Parity plot for all experimental data (circles) using predictions from Model F for (a) $\mathrm{EO}$ formation rates and (b) $\mathrm{CO}_{2}$ formation rates. Dashed lines show parity and $20 \%$ error bounds. Experimental uncertainty in measured reaction rates are $\pm 20 \%$ of the reported values. Experimental data were previously reported in Harris and Bhan ${ }^{1}$ and Chen et al. ${ }^{2}$ 


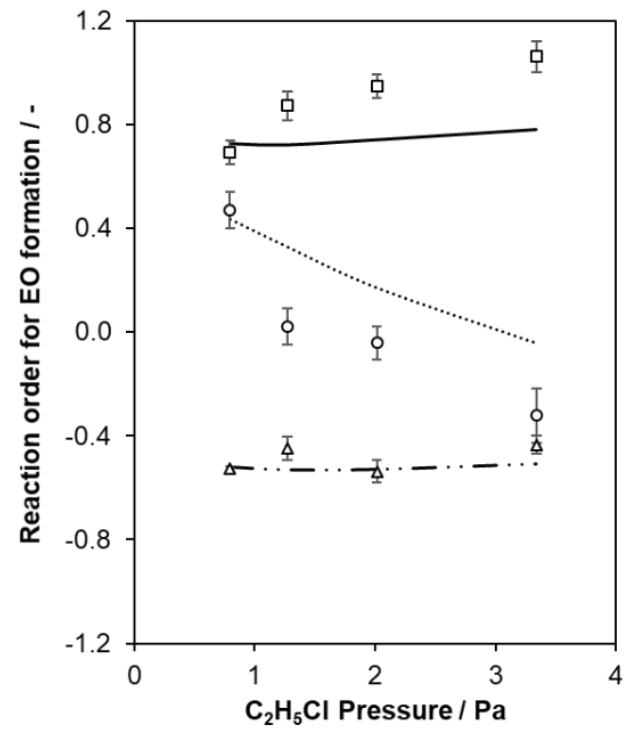

Figure S.13 Comparison of experimentally measured apparent reaction orders (symbols) with reaction orders predicted from Model F (lines) for dioxygen (squares, solid line), ethylene (circles, dashed line), and carbon dioxide (triangles, dashed-dotted line). Experimental uncertainty in measured reaction rates are $\pm 20 \%$ of the reported values. Experimental data were previously reported in Harris and Bhan ${ }^{1}$ and Chen et al. ${ }^{2}$

\section{S.2 References}

1. Harris, J. W.; Bhan, A., Moderation of chlorine coverage and ethylene epoxidation

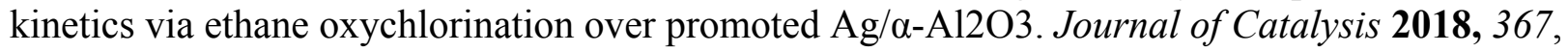
62-71.

2. Chen, C.-J.; Harris, J. W.; Bhan, A., Kinetics of ethylene epoxidation on a promoted $\mathrm{Ag} / \alpha-\mathrm{A} 12 \mathrm{O} 3$ catalyst - the effects of product and chloride co-feeds on rates and selectivity. Chemistry A European Journal 2018, 24, 12405-12415. 